\title{
Possible Role of Single Stranded DNA Binding Protein 3 on Skin Hydration by Regulating Epidermal Differentiation
}

\author{
Mi-Ra Choi*, Jung-Min Shin*, Young-Ah Shin ${ }^{1}$, Yun-Hee Chang ${ }^{2}$, Min-Youl Chang ${ }^{3}$, Cho-Ah Lim, \\ Kyung-Cheol Sohn, Young-Joon Seo, Chang-Deok Kim, Jeung-Hoon Lee, Young Lee \\ Department of Dermatology, College of Medicine, Chungnam National University, Daejeon, ${ }^{1}$ ichrogene, Suwon, ${ }^{2}$ LG Household and \\ Healthcare, Daejeon, ${ }^{3}$ Department of Bio-Cosmetic Science, Seowon University, Cheongju, Korea
}

Background: Skin hydration is a common problem both in elderly and young people as dry skin may cause irritation, dermatological disorders, and wrinkles. While both genetic and environmental factors seem to influence skin hydration, thorough genetic studies on skin hydration have not yet been conducted. Objective: We used a genome-wide association study (GWAS) to explore the genetic elements underlying skin hydration by regulating epidermal differentiation and skin barrier function. Methods: A GWAS was conducted to investigate the genetic factors influencing skin hydration in 100 Korean females along with molecular studies of genes in human epidermal keratinocytes for functional study in vitro. Results: Among several single nucleotide polymorphisms identified in GWAS, we focused on Single Stranded DNA Binding Protein 3 (SSBP3) which is associated with DNA replication and DNA damage repair. To better understand the role of SSBP3 in skin cells, we introduced a calcium-induced differentiation keratinocyte culture system model and found that SSBP3 was upregulated in keratinocytes in a differ-

Received November 20, 2017, Revised March 22, 2018, Accepted for publication March 26, 2018

*These authors have equally contributed to the article.

Corresponding author: Cho-Ah Lim, Department of Dermatology, College of Medicine, Chungnam National University, 266 Munhwa-ro, Jung-gu, Daejeon 35015, Korea. Tel: 82-42-280-7702, Fax: 82-42-280-7932, E-mail: ttole17@naver.com

ORCID: https://orcid.org/0000-0001-9084-3906

This is an Open Access article distributed under the terms of the Creative Commons Attribution Non-Commercial License (http://creativecommons. org/licenses/by-nc/4.0) which permits unrestricted non-commercial use, distribution, and reproduction in any medium, provided the original work is properly cited.

Copyright $₫$ The Korean Dermatological Association and The Korean Society for Investigative Dermatology entiation dependent manner. When SSBP3 was overexpressed using a recombinant adenovirus, the expression of differentiation-related genes such as loricrin and involucrin was markedly increased. Conclusion: Taken together, our results suggest that genetic variants in the intronic region of SSBP3 could be determinants in skin hydration of Korean females. SSBP3 represents a new candidate gene to evaluate the molecular basis of the hydration ability in individuals.

(Ann Dermatol 30(4) 432 440, 2018)

\section{-Keywords-}

Cell differentiation, Genome-wide association study, Hydration, Keratinocytes, Single stranded DNA binding protein 3

\section{INTRODUCTION}

Skin dryness, xerosis, is a common problem not only in the elderly but also in various skin disorders. Xerosis can be caused by numerous factors such as dysregulation of epidermal differentiation and lipid content, the use of drugs such as diuretics and overuse of heaters or air conditioners ${ }^{1}$. Skin xerosis causes pruritus, leading to skin inflammation, infections, and even wrinkles. Therefore, research to find active molecules to enhance skin hydration by normalizing epidermal differentiation and barrier function is of great interest.

The epidermis is the outermost cellular layer of skin, which provides functions such as the protection of the body from environmental challenges, prevention of water loss, and thermoregulation. The barrier function of the epidermis involves the expression of numerous tissue-specific genes, most of which are specifically expressed in late ker- 
atinocyte differentiation. In order to establish proper skin barrier, keratinocytes undergo a complex epidermal differentiation process, which is the transition from basal keratinocytes to corneocytes ${ }^{2}$. The dysregulation of keratinocyte differentiation results in skin barrier disruption and causes skin dryness and skin diseases such as psoriasis and atopic dermatitis ${ }^{3}$.

Recently, there have been many studies investigating the genetic variants in skin phenotypes using a genome-wide association study (GWAS), but most GWAS on skin phenotypes have only focused on the pigmentation traits or tanning abilities between races ${ }^{4-6}$; there have been no previous reports of GWAS on skin hydration. In the present study, we performed a GWAS to determine the genetic factors influencing skin hydration in Korean females, and we define the functional roles of genes containing selected single nucleotide polymorphisms (SNPs) in human epidermal keratinocytes.

\section{MATERIALS AND METHODS}

\section{Population and phenotype}

The local Ethics Committee and the Institutional Review Board (IRB) at Chungnam University Hospital (IRB no. 2012-08-019) approved this study, and informed consent was obtained from all participants regarding the study's scientific aims as well as the use of saliva, DNA and clinical records for all stages. A total of 100 healthy volunteers were enrolled for the study (Korean females, aged 30 50 years) living in the same city. Skin capacitance, a widely accepted parameter of stratum corneum hydration, was measured using a Corneometer ${ }^{\circledR}$ CM 825 (Courage \& Khazaka Electronic, Cologne, Germany) on the anterior aspect of left cheek. All measurement were done after sit and rest for $15 \mathrm{~min}$ in constant temperature and humidity conditions $\left(22^{\circ} \mathrm{C} \pm 2{ }^{\circ} \mathrm{C}, 50 \% \pm 5 \%\right)$. The tested volunteers were divided into groups according to the corneometer (CM) value: i) $C M$ value $\leq 40$ (dry skin), ii) $40<\mathrm{CM}$ value $<60$, and iii) CM value $\geq 60$ (hydrated skin) (Table 1).

\section{Genotyping, quality control and GWAS}

The Oragene (DNA Genotek, Kanata, ON, Canada) saliva kit was used to collect highly purified DNA from the saliva of volunteers. In the GWAS, 100 subjects were genotyped by using the Axio $^{\text {TM }}$ Genome-Wide ASI 1 Array Plate (Affymetrix, Santa Clara, CA, USA) comprised of 598,100 SNPs. The PLINK (ver. 1.06) and R statistical programs (http://www.r-project.org/) (ver. 2.10.1) were used for quality control procedures. SNPs were filtered if the following criteria were satisfied: i) a call rate of $<95 \%$ $(n=15,314)$, ii) a minor allele frequency of $<1 \%$
( $\mathrm{n}=76,211)$, and iii) a significant deviation from HardyWeinberg equilibrium of $\leq 1 \times 10^{-3}(\mathrm{n}=489)$. After quality control, 506,945 SNPs $(84.7 \%$ of 598,375$)$ were remaining for association analysis. Association analysis was performed using 506,945 common SNPs that satisfied the quality control criteria using quantitative trait analysis (Wald test).

\section{Immunohistochemical staining}

Skin samples were obtained from 5 patients from skin lesions in trunk of patients with psoriasis, atopic dermatitis or ichthyosis. The tissues were fixed in $10 \%$ formalin for $24 \mathrm{~h}$ and embedded in paraffin. Sections of skin specimens were dewaxed, rehydrated, and then washed three times with phosphate-buffered saline. After treatment with proteinase $\mathrm{K}(1 \mathrm{mg} / \mathrm{ml})$ for $5 \mathrm{~min}$ at $37^{\circ} \mathrm{C}$, sections were treated with $\mathrm{H}_{2} \mathrm{O}_{2}$ for 10 min at room temperature, placed in a blocking-solution (Dako, Carpinteria, CA, USA) for 20 min, followed by reaction with the appropriate primary antibodies. Sections were incubated sequentially with peroxidase-conjugated secondary antibodies (Upstate, LakePlacid, NY, USA) and visualized using a Chemmate Envision Detection Kit (Dako). Following antibodies were used in this study: single stranded DNA binding protein 3 (SSBP3) (Abcam, Cambridge, MA, USA). The intensity of SSBP3 in epidermis was analyzed using ImageJ analysis program (http://imagej.nih.gov/ij/docs/index.html).

\section{Cell culture}

Human skin tissues were obtained under the written informed consent of donors, in accordance with the ethical committee approval process of the Institutional Review Board of Chungnam National University Hospital (IRB no. 2016-07-009). Human skin tissues were obtained from trunk skin that was the remnant of benign skin tumor

Table 1. Characteristics of enrolled subjects

\begin{tabular}{lc}
\hline \multicolumn{1}{c}{ Characteristic } & GWAS \\
\hline No. of subjects & 100 \\
Chronologic age $(\mathrm{yr})$ & $40.0 \pm 4.7$ \\
Positive smoking history (\%) & 0 \\
Personal history of skin cancer (\%) & 0 \\
Skin hydration* $(\mathrm{n}=100)$ & $58.01 \pm 16.85$ \\
$\mathrm{CM}$ value $\geq 60(\mathrm{n}=52)$ & $73.58 \pm 4.14$ \\
$40<$ CM value $<60(\mathrm{n}=32)$ & $43.90 \pm 2.23$ \\
$\mathrm{CM}$ value $\leq 40(\mathrm{n}=16)$ & $35.62 \pm 2.77$ \\
\hline
\end{tabular}

Values are presented as number only, mean \pm standard deviation, or \%. GWAS: genome-wide association study, CM: corneometer. *Capacitance a.u. measured by Corneometer ${ }^{\mathbb{R}}$ CM 825 (Courage \& Khazaka Electronic, Germany). 
surgery. Primary keratinocytes were cultured according to the method previously described ${ }^{7}$. Human epidermal keratinocytes were cultured in keratinocyte-serum free medium supplemented with bovine pituitary extract and recombinant human epidermal growth factor (Life Technologies Corporation, Grand Island, NY, USA).

\section{Production of recombinant adenovirus}

Total RNA was isolated from cultured skin epithelial cells using Easy-Blue RNA extraction kit (Intron, Daejeon, Korea). Two $\mu \mathrm{g}$ of total RNA was reverse transcribed with moloney-murine leukaemia virus (M-MLV) reverse transcriptase (RTase) (ELPIS Biotech, Daejeon, Korea). Aliquot of RT mixture was subjected to polymerase chain reaction (PCR) cycles with primer set for SSBP3 5'-GGCAGATCTA TGTTTGCCAAAGGCAAAGGC-3' and 5'-TTTGTCGACT CACACACTCATCGTCATGC-3'. SSBP3 cDNA was subcloned into pENTR/CMV-Flag vector that has attL sites for site-specific recombination with a Gateway destination vector (Invitrogen, Carlsbad, CA, USA). The replication-incompetent adenoviruses were created using Virapower adenovirus expression system (Invitrogen) according to the method previously described ${ }^{8}$. Briefly, site-specific recombination between entry vector and adenoviral destination vector was achieved by LR clonase (Invitrogen). The resulting adenoviral expression vector was then transfected into 293A cells using Lipofectamine 2000 (Invitrogen). Cells were grown until 80\% cytopathic effect was seen, then harvested for preparation of recombinant adenovirus. For microRNA (miR) specific for SSBP3, target sequences were designed using Invitrogen's RNAi Designer (http://rnaidesigner.lifetechnologies.com/rnaiexpress). The miR sequences were as follows: top strand 5'-TGCTGTTCAGAACCAGACTACAAGGTGTTTTGGCCA CTGACTGACACCTTGTACTGGTTCTGAA-3' and bottom strand 5'-CCTGTTCAGAACCAGTACAAGGTGTCAGTCA GTGGCCAAAACACCTTGTAGTCTGGTTCTGAAC-3'. The double-stranded DNA oligonucleotides were synthesized and cloned into the parental vector pcDNA6.2GW/EmGFP-miR (Invitrogen). The expression cassette for miR was moved into the pENTR/CMV vector and adenovirus was made as previously reported ${ }^{8}$.

\section{Calcium-induced differentiation model}

To induce keratinocyte differentiation in vitro, we adopted a well-established calcium-induced differentiation model. After adenoviral transduction, cells were replenished with keratinocyte-serum free medium and then cultured for a further 3 days with or without calcium (1.8 mM).

\section{Western blot analysis}

Cells were lysed in Proprep solution (Intron). Total protein was measured using a BCA protein assay kit (Pierce Biotechnology, Rockford, IL, USA). Samples were run on SDS-polyacrylamide gels, transferred to nitrocellulose membranes and incubated with appropriate antibodies. Blots were then incubated with peroxidase-conjugated secondary antibodies, visualized by enhanced chemiluminescence (Intron). For determination of secreted proteins, cell culture medium was concentrated using a Protein concentration kit (Elpis Biotech). The following primary antibodies were used in this study: SSBP3 (Abcam); actin (Sigma-Aldrich, St. Louis, MO, USA).

\section{Reverse transcription-PCR (RT-PCR)}

Total RNAs were isolated using Easy-Blue RNA extraction kit. Two $\mu \mathrm{g}$ of total RNAs were reverse transcribed with M-MLV RTase (Elpis Biotech). Aliquots of RT mixture were amplified using SYBR Green real-time PCR master mix (Elpis Biotech). The following primers sequences were used: SSBP3, 5'-ATGCTTGGGACTGGATGGAA and 5'-C AAAGACCAGCTACAGGGGA; Involucrin, CCATCAGGA GCAAATGAAACAG and GCTCGACAGGCACCTTCTG; Loricrin, AATAGATCCCCCAGGGTACCA and CGGTGCC CCTGGAAAAC; K1, GGCTATGAC ССТGCTTTGTTCT and TCATGTGGGTGGTGGTCACT; GAPDH, 5'-TGCAC CACCAACTGCTTAGC and 5'-GGCATGGACTGTGGTCA TGAG.

\section{Luciferase assay}

Cells were grown at $70 \%$ confluency in a 24 well culture plate and co-transduced with reporter adenovirus and SSBP3 expressing adenovirus. After adenoviral transduction, cells were replenished with fresh medium. Cells were further incubated for $48 \mathrm{~h}$, and then cellular extracts were prepared using cell lysis buffer. Luciferase activities were determined using Luciferase assay system (Promega, Madison, WI, USA), according to the recommended protocol.

\section{Statistical analysis for in vitro study}

RT-PCR and luciferase assay were evaluated statistically using one-way ANOVA using IBM SPSS Statistics software (ver. 22.0; IBM Co., Armonk, NY, USA). Statistical significance was set at $p<0.01$.

\section{RESULTS}

\section{Association between SNPs and hydration phenotypes}

To identify significant loci associated with skin hydration, 
we genotyped 598,100 SNPs in 100 individuals using Axiom $^{\text {TM }}$ Genome-Wide ASI 1 Array Plate. The samples consisted of individuals with CM values. After quality control, we analyzed the association of 501,262 common markers using quantitative trait analysis (Wald test). Among several SNPs identified in GWAS, we focused on SSBP3 which is known to be associated with DNA replication, DNA damage repair and cell differentiation (Table 2).

\section{Expression of SSBP3 in epidermal keratinocytes}

First, we evaluated the endogenous expression of SSBP3 in epidermal cells, including normal human epidermal keratinocytes (NHEKs) and HaCaT cells, and found that SSBP3 was expressed in both skin cell lines (Fig. 1A). Next, we confirmed the expression of SSBP3 in normal human skin using immunohistochemical analysis. We noted that SSBP3 is expressed in all layers of epidermis. However, the intensity of SSBP3 was low in proliferative cells in the basal cell layer of epidermis but strongest in the spinous layers which begin to express proteins required for cornification (Fig. 1B).

\section{Overexpression of SSBP3 promotes keratinocyte differentiation}

Since the expression of SSBP3 was increased during the keratinocyte differentiation process, we investigated the putative role of SSBP3 in epidermal differentiation. To examine SSBP3 during keratinocyte differentiation, we adopted a well-established calcium-induced differentiation model. As expected, SSBP3 was upregulated in a time-dependent manner from day 3 after calcium treatment and decreased at day 7, which was consistent with the result of immunohistochemical analysis showing that SSBP3 was most prominent in the spinous layer of the epidermis (Fig. 2A). We then constructed a recombinant adenovirus expressing SSBP3 and transduced cultured human epidermal keratinocytes (Fig. 2B). Overexpression of SSBP3 led to a significant increase in mRNA levels of epidermal differentiation related markers such as $\mathrm{K} 1$, involucrin, and loricrin (Fig. 2C). Next, we transduced keratinocytes with each involucrin-luc and loricrin-luc reporter adenoviruses, in which about $3.7 \mathrm{~kb}$ of the involucrin promoter fragment and $2.0 \mathrm{~kb}$ of the loricrin promoter fragment were fused to

Table 2. SSBP gene family identified in the GWAS

\begin{tabular}{lcccccccc}
\hline rs no. NCBI* & Chr. & Position & Function & Genes & No. of samples & $\beta$ & SE & $p$-value \\
\hline rs12041903 & 1 & 54772434 & Intron & SSBP3 & 100 & -9.53 & 3.469 & 0.007156 \\
rs7523947 & 1 & 54766816 & Intron & SSBP3 & 99 & -7.952 & 3.022 & 0.009887 \\
rs11206323 & 1 & 54762449 & Intron & SSBP3 & 99 & -5.601 & 2.545 & 0.03013 \\
rs435070 & 5 & 80981992 & Intron & SSBP2 & 97 & -4.598 & 2.143 & 0.03446 \\
rs454578 & 5 & 80966666 & Intron & SSBP2 & 98 & -4.926 & 2.33 & 0.03711 \\
\hline
\end{tabular}

GWAS: genome-wide association study, Chr.: chromosome, SE: standard error. *Single nucleotide polymorphisms on National Center for Biotechnology Information (NCBI) reference assembly.

A

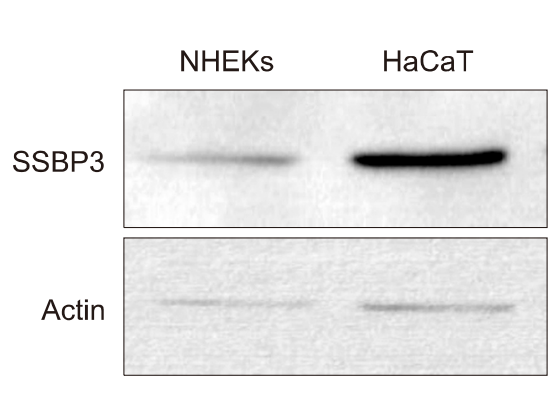

B

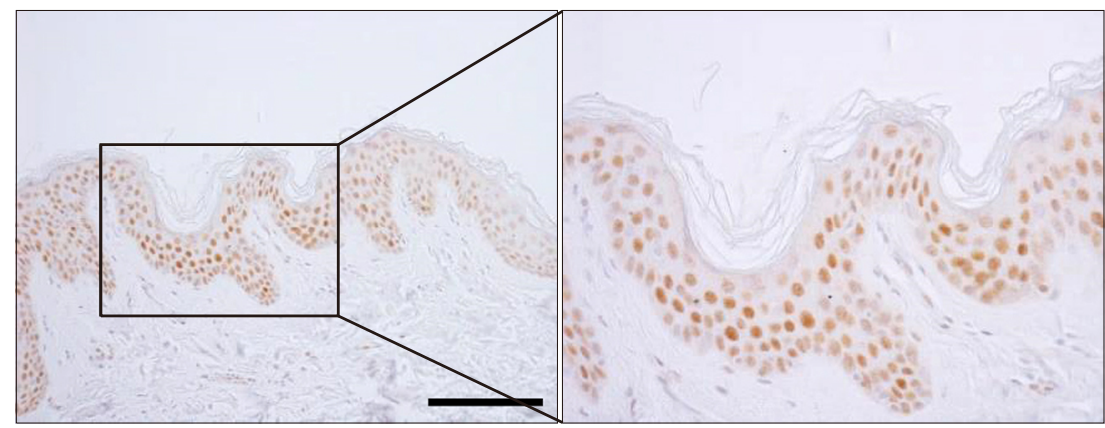

Fig. 1. Expression of SSBP3 in epidermal keratinocytes and epidermis. (A) Cellular extracts were prepared from cultured normal human epidermal keratinocytes (NHEKs) and HaCaT cells. The endogenous protein expression of SSBP3 was detected via western blot analysis. (B) Paraffin-embedded normal skin tissue was immunohistochemically stained with anti-SSBP3. Immunohistochemical staining shows nuclear SSBP3 staining in all epidermal layers and increased SSBP3 positive staining in the spinous layer (hematoxylin and DAB, $\times 200$; scale bar $=50 \mu \mathrm{m}$ ). 
A

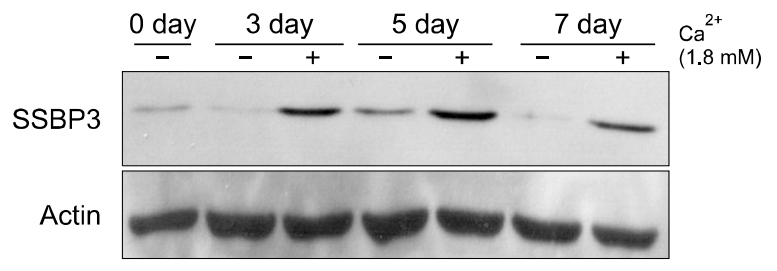

C

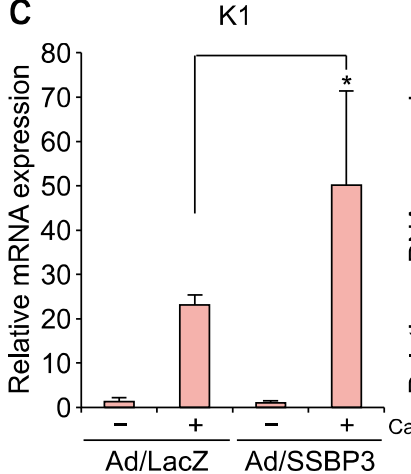

D

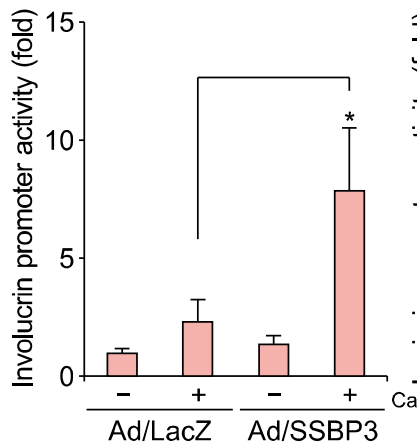

B

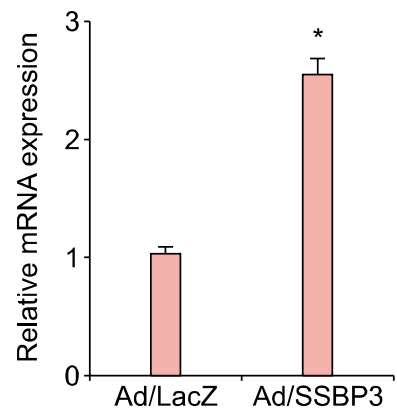

Loricrin

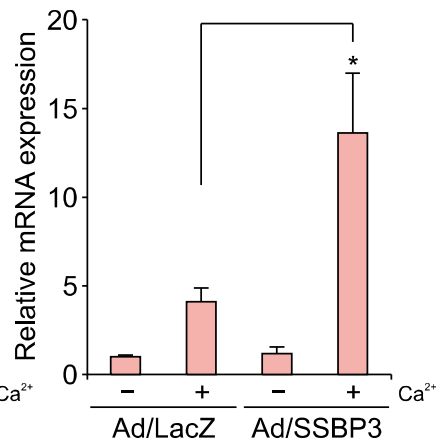

E
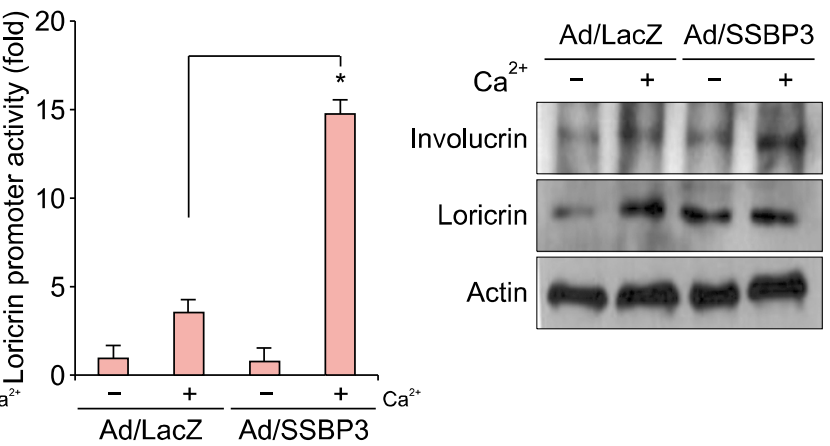

Fig. 2. Effect of SSBP3 overexpression on keratinocyte differentiation. (A) Keratinocytes were treated with $1.8 \mathrm{mM} \mathrm{CaCl}_{2}$ for indicated times. SSBP3 protein levels were detected via western blot analysis. Actin was used as a loading control. (B) Keratinocytes were transduced with recombinant adenoviruses expressing SSBP3 or LacZ control for $12 \mathrm{~h}$. Cells were replenished with fresh medium and then cultured for 3 days. mRNA level of SSBP3 was determined by quantitative polymerase chain reaction (qPCR). (C) After overexpression of SSBP3 in keratinocytes, cells were further treated with $1.8 \mathrm{mM} \mathrm{CaCl}_{2}$ for 3 days. The mRNA levels of epidermal differentiation-related markers involucrin, loricrin, and cytokeratin 1 (K1) were measured using qPCR. (D) The effect of SSBP3 overexpression on loricrin and involucrin promoter activity. The involucrin or loricrin promoter-luciferase reporter adenoviruses were co-transduced with adenoviruses expressing SSBP3 or LacZ for $12 \mathrm{~h}$. Cells were replenished with fresh medium and treated with $1.8 \mathrm{mM} \mathrm{CaCl}_{2}$. Cells were further cultured for 3 days and assayed for luciferase activity. (E) After overexpression of SSBP3 in keratinocytes, cells were further treated with $1.8 \mathrm{mM} \mathrm{CaCl}_{2}$ for 3 days. The protein expression of involucrin and loricrin were examined by western blot analysis. Actin was used as a loading control. Values are presented as mean \pm standard deviation of triplicate measurements $\left({ }^{*} p<0.01\right)$.

the luciferase gene. Overexpression of SSBP3 significantly increased the involucrin and loricrin luciferase activities compared to the Ad/LacZ control (Fig. 2D). And we also confirmed that overexpression of SSBP3 increased the expression of involucrin and loricrin in protein levels (Fig. 2E).

\section{Knockdown of SSBP3 inhibits keratinocyte differentiation}

We further confirmed the role of SSBP3 on keratinocyte differentiation by knockdown experiment. To this end, we transduced keratinocytes with recombinant adenovirus ex- 

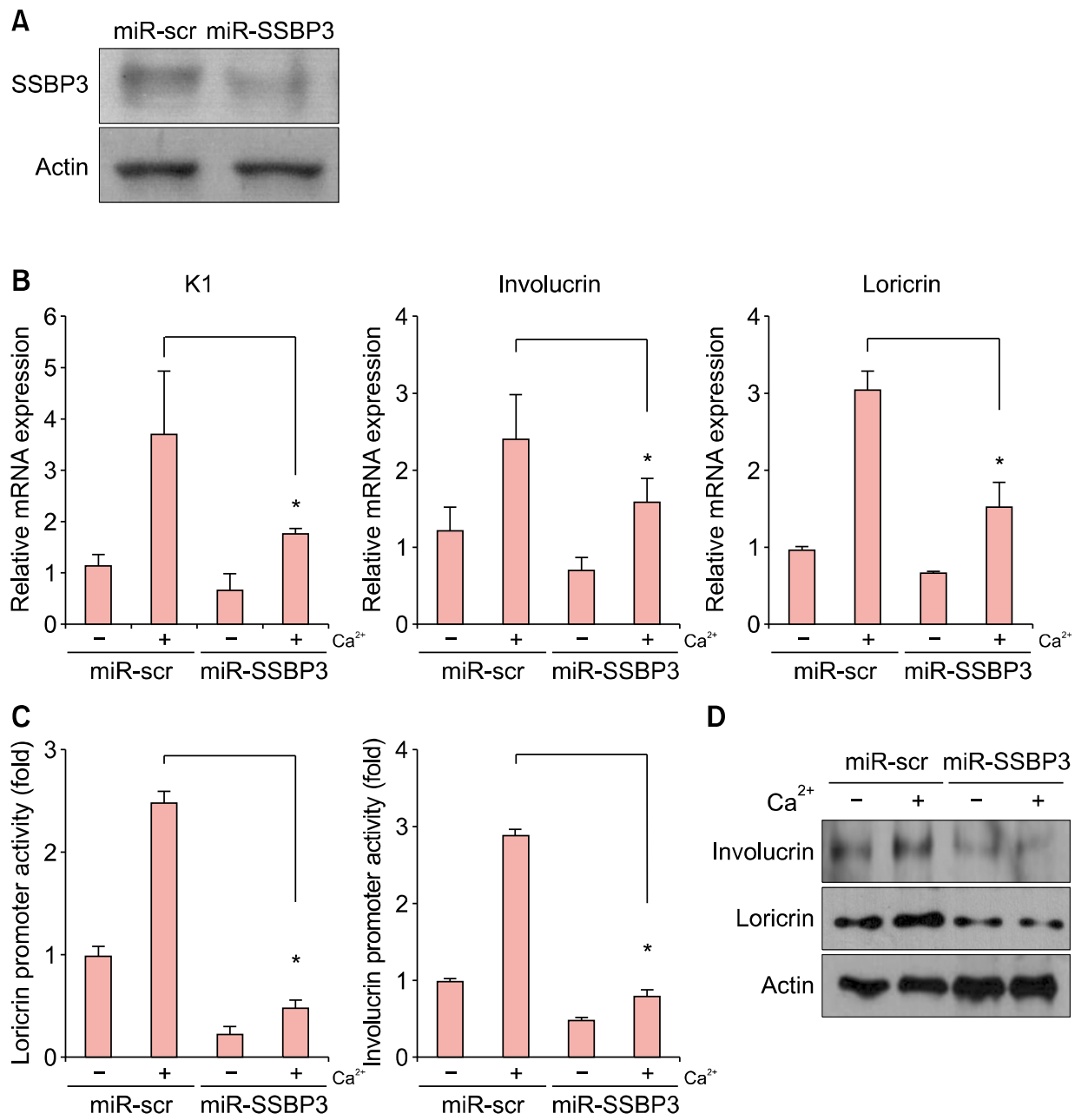

Fig. 3. Effect of SSBP3 knockdown on keratinocyte differentiation. (A) Keratinocytes were transduced with adenoviruses expressing microRNA (miR)-SSBP3 or scrambled control (miR-scr) for $12 \mathrm{~h}$. Cells were replenished with fresh medium and then cultured for 3 days. The SSBP3 protein level was measured via western blot analysis. Actin was used as the loading control. (B) After knockdown of SSBP3 in keratinocytes, cells were further treated with $1.8 \mathrm{mM} \mathrm{CaCl}_{2}$ for 3 days. The mRNA levels of epidermal differentiation-related markers involucrin, loricrin, and cytokeratin 1 (K1) were measured using quantitative polymerase chain reaction. (C) The effect of SSBP3 knockdown on involucrin and loricrin promoter activity. The involucrin or loricrin promoter-luciferase reporter adenoviruses were co-transduced with adenoviruses expressing miR-SSBP3 or miR-scr for $12 \mathrm{~h}$. Cells were replenished with fresh medium and treated with $1.8 \mathrm{mM} \mathrm{CaCl}_{2}$. Cells were further cultured for 3 days and assayed for luciferase activity. (D) After knockdown of SSBP3 in keratinocytes, cells were further treated with $1.8 \mathrm{mM} \mathrm{CaCl}_{2}$ for 3 days. The protein expression of involucrin and loricrin were examined by western blot analysis. Actin was used as a loading control. Values are presented as mean \pm standard deviation of triplicate measurements $\left({ }^{*} p<0.01\right)$.

pressing miR specific for SSBP3 (Fig. 3A). As shown in Fig. $3 B$, mRNA levels of $\mathrm{K} 1$, involucrin, and loricrin were significantly decreased by SSBP3 knockdown even with the calcium treatment. Consistent with the quantitative PCR (qPCR) analysis results, SSBP3 downregulation led to decreased luciferase activities of loricrin and involucrin compared to the control groups (Fig. 3C). The results suggest that knockdown of SSBP3 decreased differentiation of keratinocytes through decreased transcriptional activity via specific DNA binding to the promoter regions of epidermal differentiation-related genes. Knockdown of SSBP3 also inhibited the expression of involucrin and loricrin in protein levels (Fig. 3D).

\section{Expression of SSBP3 in skin diseases related to keratinocyte differentiation}

Since SSBP3 can regulate keratinocyte differentiation in vitro, we hypothesized that SSBP3 expression may have a correlation to skin disease relating to keratinocyte differentiation. We examined SSBP3 expression in ichthyosis, atopic dermatitis and psoriasis, which are skin disorders showing abnormal keratinocyte differentiation and de- 
A Psoriasis

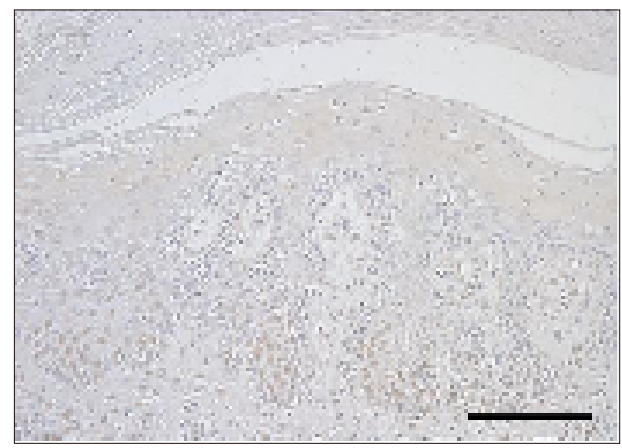

C Atopic dermatitis

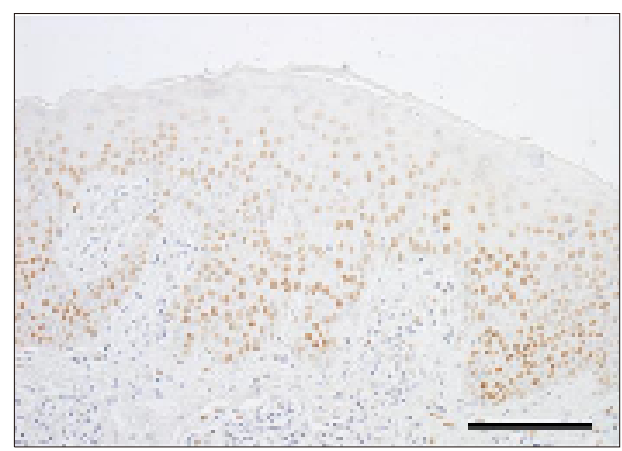

B Ichthyosis
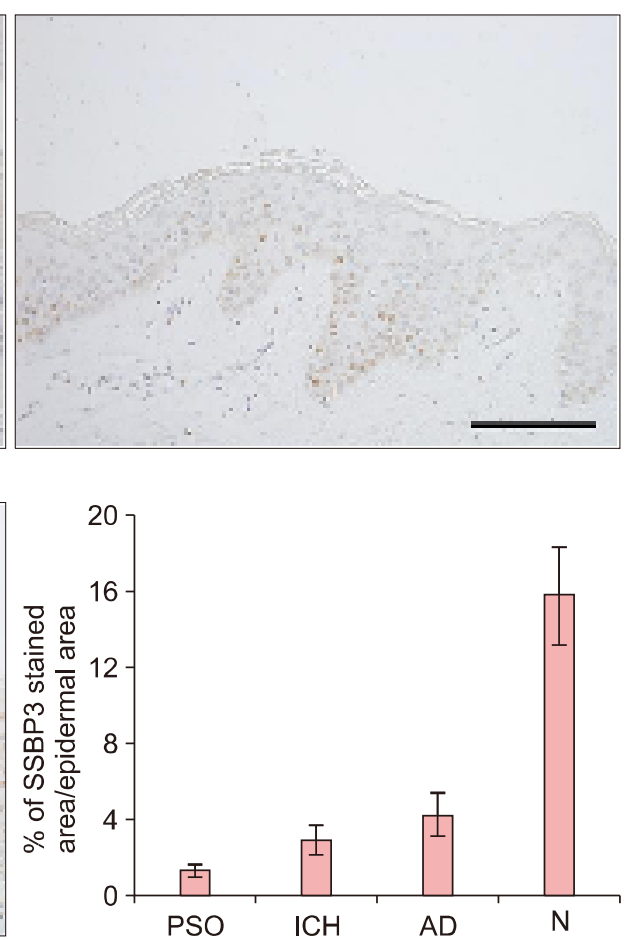

Fig. 4. Immunohistochemical analysis of SSBP3 in epidermal differentiation-related skin disorders. (A) Psoriasis, $(\mathrm{B})$ ichthyosis, and $(\mathrm{C})$ atopic dermatitis. $(A \sim C)$ Paraffin-embedded lesional tissues were immunohistochemically stained with anti-SSBP3 (hematoxylin and DAB, $\times 200$, scale bars $=50 \mu \mathrm{m})$. Positive staining of SSBP3 in epidermis was quantified. PSO: psoriasis, $\mathrm{ICH}$ : ichthyosis, $\mathrm{AD}$ : atopic dermatitis, $\mathrm{N}$ : normal skin. creased moisturization due to a defect in the skin barrier. As expected, decreased expression of SSBP3 was found in psoriasis, a multisystemic disease characterized by hyperproliferation and altered differentiation of the epidermis (Fig. 4A). SSBP3 was also decreased in the spinous layer in ichthyosis, a disease caused by a loss-of-function mutation that results in keratinocyte disruption (Fig. 4B). Finally, diffuse nuclear staining of SSBP3 was observed in the basal and lower spinous layers of epidermis in atopic dermatitis, an inflammatory skin disease showing defects in keratinocytes differentiation and the skin barrier; however, the intensity of staining was decreased in the upper spinous and granular layers (Fig. 4C).

\section{DISCUSSION}

Epidermis, the outermost part of the skin composed mainly of keratinocytes, is a self-renewing, multilayered, stratified, and keratinized squamous epithelium. It provides a physical barrier from dehydration and various environmental stresses ${ }^{2}$. To maintain the protective barrier continuously, keratinocytes should be well balanced in proliferation, differentiation and apoptosis. Incomplete barrier acquisition and dysregulation of keratinocyte differentiation manifest as dehydration, electrolyte imbalance, poor thermoregulation and predisposition to infection ${ }^{9,10}$. In addition, abnormal epidermal keratinocyte differentiation causes barrier dysfunction and skin disorders such as psoriasis and atopic dermatitis.

Decreased hydration is a problem not only in people with skin disorders, but also in healthy populations, and the frequency of dry skin increases with aging. In dry skin, there are significant changes in the epidermal expression of basal and differentiation-related keratins and premature expression of involucrin, which is related to terminal differentiation, meaning that skin hydration is closely related to epidermal differentiation ${ }^{11}$. As adequate skin hydration is critical for maintaining healthy skin, there is significant ongoing research into finding active target molecules to improve skin hydration.

Recent developments in the study of molecular genetics have added to our understanding of how genetic differences account for variations among individuals. GWAS, a method used to identify genetic variations in different individuals, focuses on associations between SNPs and phenotypes. Numerous conventional genetic studies have identified particular target regions and susceptible loci for diseases and traits. Lately, genetic studies have been conducted to find the loci associated with skin phenotypes such as pigmentation ${ }^{12-14}$. Yet, there has been no published genetic study identifying loci associated with phenotypes of skin hydration. In this report, we present GWAS analyses that demonstrate that SSBP3 is associated with an individual's hydration phenotype. In addition, we 
present a functional study of SSBP3 confirming the biological relevance of this gene in skin hydration. We first selected all the genes that show the relationship with cell differentiation signaling pathway but not yet been investigated in epidermal differentiation. The lowest $p$-value of the gene SSBP3 is $7.15 \times 10^{-3}$ in the current study, which does not reach GWAS significant level but several SNPS were identified in the intronic region of SSBP3 and SSBP2. Also, previous literatures elucidated the functional role of SSBP3 in cell differentiation and cell cycle; however, no studies have reported a relationship between SSBP3 and epidermal keratinocytes. So, we presented other evidences to support the GWAS through further functional studies that SSBP3 has susceptibility of epidermal differentiation using in vitro cell experiments.

SSBPs are part of the oligonucleotide/oligosaccharidebinding fold domain family and function in DNA replication at the restart of stalled replication forks, DNA damage repair, cell cycle-checkpoint activation, and telomere maintenance $^{15}$. The human SSBP3 gene was first identified as a member of an evolutionarily conserved and ubiquitously expressed gene family with possible involvement in cancer $^{16}$. SSBP3 has also been reported to have important roles in neuronal morphology and is highly expressed in skeletal muscle, heart, brain, kidney, and hematopoietic tissues. Previous studies have shown that SSBPs modulate axis formation in Xenopus, wing development in Drosophila, and head morphogenesis in mice ${ }^{17-20}$. SSBP3 has been found to regulate DNA replication, recombination, and repair by binding to DNA, and overexpression of SSBP3 induces differentiation of mouse embryonic stem cells into trophoblast-like cells, indicating the possibility that SSBP3 might act as a positive regulator in epidermal differentiation ${ }^{21,22}$. In this study, all the genetic variants were detected in the intronic region of SSBP3. In the past, introns are often considered as useless part of the gene that is removed to create an mRNA. However, recent papers propose the effect of introns on expression of genes. The mechanism of how introns regulate gene expression is not entirely clear, but many studies show introns can affect gene expression at many different levels, including transcription, polyadenylation, mRNA export, translational efficiency, and the rate of mRNA decay ${ }^{23,24}$.

In conclusion, we demonstrated a role for SSBP3 in keratinocyte differentiation using an adenoviral gene delivery system. Specifically, SSBP3 overexpression in keratinocytes led to a significant increase in epidermal differentiationrelated gene expression and activity. Conversely, knockdown of SSBP3 decreased epidermal differentiation-related gene expression and activity. In addition, psoriasis and atopic dermatitis, which are the skin disorders with altered epidermal differentiation and skin dehydration, showed decreased expression of SSBP3 ${ }^{25}$. These results suggest that SSBP3 may be linked to an individual's hydration skin phenotype by positively regulating keratinocyte differentiation. Although the number of enrolled subjects was relatively small, and the $p$-value of the SNP was not small enough to confirm the relationship between the SNP and skin hydration, we believe these results may contribute to a better understanding of skin hydration through better understanding of the factors regulating epidermal differentiation and provide new insights into the development of possible treatment targets for abnormal differentiationrelated skin disorders via modulation of SSBP3. A larger sample size would be useful in detecting additional SNPs associated with skin hydration in future studies.

\section{ACKNOWLEDGMENT}

This study was supported by a grant of the Korean Health Technology R\&D Project, Ministry of Health \& Welfare, Republic of Korea (grant no. HN10C0013).

\section{CONFLICTS OF INTEREST}

The authors have nothing to disclose.

\section{REFERENCES}

1. White-Chu EF, Reddy M. Dry skin in the elderly: complexities of a common problem. Clin Dermatol 2011;29:37-42.

2. Kalinin AE, Kajava AV, Steinert PM. Epithelial barrier function: assembly and structural features of the cornified cell envelope. Bioessays 2002;24:789-800.

3. Lebwohl M, Herrmann LG. Impaired skin barrier function in dermatologic disease and repair with moisturization. Cutis 2005;76(6 Suppl):7-12.

4. Candille SI, Absher DM, Beleza S, Bauchet M, McEvoy B, Garrison NA, et al. Genome-wide association studies of quantitatively measured skin, hair, and eye pigmentation in four European populations. PLoS One 2012;7:e48294.

5. Zhang $M$, Song $F$, Liang L, Nan $H$, Zhang J, Liu $H$, et al. Genome-wide association studies identify several new loci associated with pigmentation traits and skin cancer risk in European Americans. Hum Mol Genet 2013;22:2948-2959.

6. Nan H, Kraft P, Qureshi AA, Guo Q, Chen C, Hankinson $\mathrm{SE}$, et al. Genome-wide association study of tanning phenotype in a population of European ancestry. J Invest Dermatol 2009; 129:2250-2257.

7. Lee JS, Kim DH, Choi DK, Kim CD, Ahn GB, Yoon TY, et al. Comparison of gene expression profiles between keratinocytes, melanocytes and fibroblasts. Ann Dermatol 2013; 25:36-45.

8. Shi G, Sohn KC, Kim SY, Ryu EK, Park YS, Lee Y, et al. Sox9 increases the proliferation and colony-forming activity of 
outer root sheath cells cultured in vitro. Ann Dermatol 2011;23:138-143.

9. Kalia YN, Nonato LB, Lund CH, Guy RH. Development of skin barrier function in premature infants. J Invest Dermatol 1998;111:320-326.

10. Sevilla LM, Nachat R, Groot KR, Klement JF, Uitto J, Djian $P$, et al. Mice deficient in involucrin, envoplakin, and periplakin have a defective epidermal barrier. J Cell Biol 2007; 179:1599-1612.

11. Engelke $M$, Jensen JM, Ekanayake-Mudiyanselage S, Proksch E. Effects of xerosis and ageing on epidermal proliferation and differentiation. Br J Dermatol 1997;137:219-225.

12. Han J, Kraft P, Nan H, Guo Q, Chen C, Qureshi A, et al. A genome-wide association study identifies novel alleles associated with hair color and skin pigmentation. PLoS Genet 2008;4:e1000074.

13. Rees JL. The genetics of sun sensitivity in humans. Am J Hum Genet 2004;75:739-751.

14. Kwak TJ, Chang YH, Shin YA, Shin JM, Kim JH, Lim SK, et al. Identification of a possible susceptibility locus for UVBinduced skin tanning phenotype in Korean females using genomewide association study. Exp Dermatol 2015;24: 942-946.

15. Ashton NW, Bolderson E, Cubeddu L, O'Byrne KJ, Richard DJ. Human single-stranded DNA binding proteins are essential for maintaining genomic stability. BMC Mol Biol 2013;14:9.

16. Castro P, Liang H, Liang JC, Nagarajan L. A novel, evolutionarily conserved gene family with putative sequencespecific single-stranded DNA-binding activity. Genomics 2002;80:78-85.

17. Chen L, Segal D, Hukriede NA, Podtelejnikov AV, Bayarsaihan D, Kennison JA, et al. Ssdp proteins interact with the LIM-domain-binding protein Ldb1 to regulate development. Proc Natl Acad Sci U S A 2002;99:14320-14325.

18. van Meyel DJ, Thomas JB, Agulnick AD. Ssdp proteins bind to LIM-interacting co-factors and regulate the activity of LIM-homeodomain protein complexes in vivo. Development 2003;130:1915-1925.

19. Nishioka N, Nagano S, Nakayama R, Kiyonari H, ljiri T, Taniguchi K, et al. Ssdp1 regulates head morphogenesis of mouse embryos by activating the Lim1-Ldb1 complex. Development 2005;132:2535-2546.

20. Enkhmandakh B, Makeyev AV, Bayarsaihan D. The role of the proline-rich domain of Ssdp1 in the modular architecture of the vertebrate head organizer. Proc Natl Acad Sci U S A 2006;103:11631-11636.

21. Cai $Y, X u$ Z, Nagarajan L, Brandt SJ. Single-stranded DNAbinding proteins regulate the abundance and function of the LIM-homeodomain transcription factor LHX2 in pituitary cells. Biochem Biophys Res Commun 2008;373:303-308.

22. Liu J, Luo X, Xu Y, Gu J, Tang F, Jin Y, et al. Single-stranded DNA binding protein Ssbp3 induces differentiation of mouse embryonic stem cells into trophoblast-like cells. Stem Cell Res Ther 2016;7:79.

23. Moabbi AM, Agarwal N, El Kaderi B, Ansari A. Role for gene looping in intron-mediated enhancement of transcription. Proc Natl Acad Sci U S A 2012;109:8505-8510.

24. Nott A, Meislin SH, Moore MJ. A quantitative analysis of intron effects on mammalian gene expression. RNA 2003;9: 607-617.

25. Lee Y, Je YJ, Lee SS, Li ZJ, Choi DK, Kwon YB, et al. Changes in transepidermal water loss and skin hydration according to expression of aquaporin-3 in psoriasis. Ann Dermatol 2012;24:168-174. 